\title{
Route of primary HTLV-1 infection regulates HTLV-1 distribution in reservoir organs of infected mice
}

\author{
MASAKAZU TANAKA ${ }^{1,2,3^{*}}$, TAKAYUKI NITTA ${ }^{1,2^{*}}$, BINLIAN SUN $^{2}$, \\ JUN-ICHI FUJISAWA ${ }^{3}$ and MASANAO MIWA ${ }^{1,2}$
}

\begin{abstract}
${ }^{1}$ Department of Genetics and Cell Biology, Graduate School of Bioscience, Nagahama Institute of Bio-Science and Technology, Nagahama, Shiga 526-0829; ${ }^{2}$ Graduate School of Comprehensive Human Sciences, University of Tsukuba, Tsukuba, Ibaraki 305-8575; ${ }^{3}$ Department of Microbiology, Kansai Medical University, Moriguchi, Osaka 570-8506, Japan
\end{abstract}

Received October 6, 2010; Accepted November 18, 2010

DOI: $10.3892 / \mathrm{etm} .2010 .179$

\begin{abstract}
Human T-cell leukemia virus type-1 (HTLV-1) causes adult T-cell leukemia and HTLV-1-associated myelo-pathy/ tropical spastic paraparesis. HTLV-1 is mainly transmitted through blood transfusion and breastfeeding, but viral proliferation in the body in vivo shortly after transmission is not well understood. To investigate whether the route of infection influences the early stages of viral proliferation, we inoculated BALB/c mice with MT-2 cells, an HTLV-1-producing human T-cell line, via different routes, and evaluated the proviral load and humoral immune responses. One month after infection, the provirus was detected in most organs of the mice infected intraperitoneally, and substantial proviral loads were detected in the peripheral blood and secondary lymphoid organs. In contrast, the mice infected intravenously and orally showed low proviral loads, and the provirus distribution was limited to the spinal cord among the intravenously inoculated mice and to the liver among the perorally inoculated mice. Mice infected intraperitoneally also exhibited higher interleukin- 2 production than the mice infected intravenously or orally, or than the uninfected control mice, while anti-HTLV-1 antibody titers were comparable between the mice infected intraperitoneally and intravenously. These results demonstrate that the route of primary HTLV-1 infection influences the establishment of HTLV-1-infected cell proliferation and the cell reservoir in mice.
\end{abstract}

\section{Introduction}

Human T-cell leukemia virus type 1 (HTLV-1) causes various diseases such as adult T-cell leukemia/lymphoma (ATL),

Correspondence to: Professor Masanao Miwa, Graduate School of Bioscience, Nagahama Institute of Bio-Science and Technology, Nagahama, Shiga 526-0829, Japan

E-mail: m_miwa@nagahama-i-bio.ac.jp

*Contributed equally

Key words: human T-cell leukemia virus type-1, mouse model, route of infection
HTLV-1-associated myelopathy/tropical spastic paraparesis (HAM/TSP), uveitis and arthropathy (1). HTLV-1 transmission mainly occurs through blood transfusion, sexual contact, breastfeeding or intrauterine transfer (2). However, not all individuals exposed to HTLV-1 develop persistent infection. Previous reports have indicated that approximately $60 \%$ of individuals who received infected blood or blood components and $15-30 \%$ of breast-fed children born to infected mothers developed HTLV-1 infection (3). In the early phase of infection, patients hold only a small percentage of HTLV-1 provirus loads in peripheral blood. In asymptomatic carriers, proviral load is maintained for a long period, and it is currently difficult to eradicate the infected cells. Importantly, high proviral loads in asymptomatic carriers are associated with the onset of ATL (4) and the progression of motor disability associated with HAM/TSP (5). In addition, a high level of HTLV-1 provirus load in circulating lymphocytes of HTLV-1 carriers is a risk factor for HTLV-1-related diseases (6-8). However, the lack of knowledge of early events of HTLV-1 spread in vivo has hindered an understanding of viral infections.

We previously inoculated mice intraperitoneally with MT-2 cells and reported that HTLV-1 provirus was integrated into the mouse genome, and that HTLV-1-infected mouse cells were distributed in various organs in the mice (9-12). The aim of the present study was to examine whether the route of viral infection affects HTLV-1 proliferation and host immune response after primary $\mathrm{HTLV}-1$ infection.

\section{Materials and methods}

Cells and animals. MT-2 cells, an HTLV-1-infected human T-cell line (13), were cultured in RPMI-1640 medium supplemented with $10 \%$ fetal calf serum. Balb/c mice were purchased from Clea, Inc., Tokyo, Japan. At 4 weeks of age, the mice were inoculated intraperitoneally, intravenously or perorally with $10^{6}$ or $10^{7}$ MT- 2 cells. The experiments were conducted in accordance with the Regulations on Animal Experiments of the University of Tsukuba, and were approved by the University's Animal Experiment Committee.

Quantification of HTLV-1 proviral load. qPCR conditions were as described previously (9). Briefly, the number of tax 
Table I. Proviral load in organs from BALB/c mice infected with HTLV-1 intraperitoneally, intravenously or perorally.

\begin{tabular}{|c|c|c|c|c|c|}
\hline \multirow[b]{2}{*}{ Organs } & \multicolumn{2}{|c|}{$\mathrm{PO}^{\mathrm{a}}$} & \multirow{2}{*}{$\frac{\mathrm{IV}^{\mathrm{a}}}{10^{6}(\mathrm{n}=5)^{\mathrm{b}}}$} & \multicolumn{2}{|c|}{$\mathrm{IP}^{\mathrm{a}}$} \\
\hline & $10^{6}(\mathrm{n}=6)^{\mathrm{b}}$ & $10^{7}(\mathrm{n}=6)^{\mathrm{b}}$ & & $10^{6}(\mathrm{n}=4)^{\mathrm{b}}$ & $10^{7}(\mathrm{n}=5)^{\mathrm{b}}$ \\
\hline PBMC & 0 & 0 & 0 & $25.6 \pm 12.1$ & $20.4 \pm 8.9$ \\
\hline Brain & 0 & 0 & 0 & $1.3 \pm 3.1$ & $0.3 \pm 0.4$ \\
\hline Salivary lymph nodes & 0 & 0 & 0 & $7.4 \pm 3.5$ & $14.4 \pm 8.4$ \\
\hline Lung & 0 & 0 & 0 & $9.0 \pm 3.3$ & $13.7 \pm 9.2$ \\
\hline Thymus & 0 & 0 & 0 & $2.1 \pm 2.4$ & $2.7 \pm 2.9$ \\
\hline Spleen & 0 & 0 & 0 & $21.9 \pm 8.1$ & $56.1 \pm 29.0$ \\
\hline Mesenteric lymph nodes & 0 & 0 & 0 & $38.9 \pm 9.3$ & $38.0 \pm 16.1$ \\
\hline Peyer's patches & 0 & 0 & 0 & $41.7 \pm 10.2$ & $78.8 \pm 25.8$ \\
\hline Liver & 0 & $1.0 \pm 1.3$ & 0 & 0 & $7.0 \pm 6.7$ \\
\hline Kidney & 0 & $0.1 \pm 0.1$ & 0 & $4.8 \pm 2.0$ & $7.8 \pm 4.9$ \\
\hline Ovary & 0 & 0 & 0 & $5.1 \pm 2.1$ & $9.1 \pm 7.8$ \\
\hline Spinal cord & 0 & 0 & $2.5 \pm 2.6$ & $0.9 \pm 2.3$ & $0.1 \pm 0.1$ \\
\hline Submandibular glands & 0 & 0 & 0 & $0.9 \pm 1.4$ & $0.8 \pm 1.3$ \\
\hline
\end{tabular}

aProviral load in the various organs was examined 1 month post-infection. Values reflect the average number of $\operatorname{tax}$ molecules per $10^{5}$ cells $\pm \mathrm{SD}$. PO, peroral inoculation; IV, intravenous inoculation; IP, intraperitoneal inoculation; PBMC, peripheral blood mononuclear cells. ${ }^{\text {bValues reflect }}$ the number of MT-2 cells introduced into the 4-week-old BALB/c mice. The number of mice examined is indicated in parentheses.

(viral gene) and mouse c-myc molecules (cellular gene control) were quantified using real-time PCR, and the HTLV-1 proviral load per $10^{5}$ mouse cells was calculated as follows: (number of tax molecules/number of mouse $c$-myc molecules/2) x $10^{5}$. Proviral load was defined as zero when there was no tax signal after 50 cycles of PCR amplification under conditions in which mouse $c$-myc was amplified.

Detection of HTLV-1 antibody in serum. The antibodies against HTLV-1 proteins in the plasma were assayed with a particle agglutination kit (Serodia HTLV-1; Fujirebio, Japan).

Interleukin-2 (IL-2) production assay. Splenic T cells from native and HTLV-1-infected mice were cultured with anti-CD3 for 2 days. The concentrations of IL- 2 in the supernatants were measured by the enzyme-linked immunosorbent assay (ELISA; Ready-SET-Go! kits, eBioscience, San Diego, CA) according to the manufacturer's instructions. The ELISA assay was specific for mouse-derived IL-2, since no cross-reactivity with human IL-2 from MT-2 cells was observed.

Statistical analysis. Welch's t-test was used for statistical analysis.

\section{Results}

Tissue distribution of the HTLV-1 provirus by various routes. As shown in Table I, in mice infected intraperitoneally with $10^{6}$ MT-2 cells, provirus was detected in most organs, and high proviral loads were detected in peripheral blood mononuclear cells, spleen and gut-associated lymphoid tissues, consistent with our previous results (12). Among mice inoculated intraperitoneally with different amounts of MT-2 cells, no significant differences in organ distribution were observed, while the mice inoculated with $10^{7}$ MT-2 cells had higher proviral loads than those inoculated with $10^{6}$ MT-2 cells. In contrast to intraperitoneal infection, intravenous or oral inoculation resulted in low proviral loads and restricted organ distribution of infected cells. Among mice infected intravenously, provirus was detected only in the spinal cord, while among mice inoculated orally with $10^{7}$ MT-2 cells, provirus was detected in the kidney and liver of 2 of the 5 mice examined. These results demonstrate that the route of viral exposure likely affects proviral load and viral organ distribution in mice.

Comparision of immune responses by various routes against $H T L V-1$. Antibody titers were determined in the sera of mice 1 month after infection using a particle agglutination kit. Antibody titers against HTLV-1 in the mice infected orally were below the detectable level (Fig. 1A), consistent with a previous report in which F344/N Jcl-rnu/+ rats were inoculated orally with MT-2 cells (14). The mice infected intraperitoneally and intravenously exhibited similar antiHTLV-1 titers. These results suggest that antibody titers do not correlate with proviral load in mice 1 month after infection, and corroborate similar findings in our previous report, in which various syngeneic mouse strains were used (9).

IL-2 production and proliferation of HTLV-1-infected cells. Continuous growth of HTLV-1-infected cells in vitro requires IL-2-mediated signaling (15), and IL-2 also likely drives the 
A

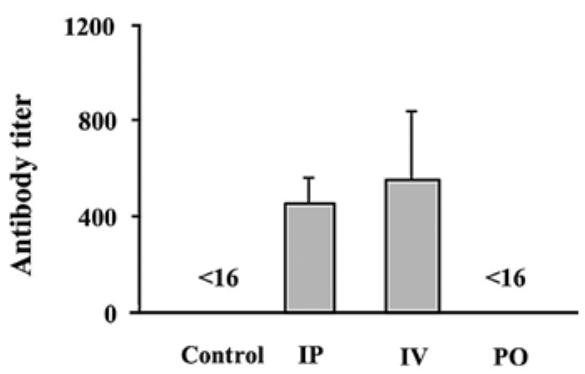

B

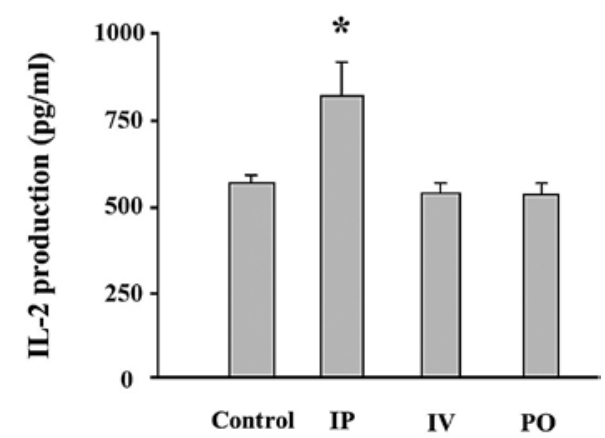

Figure 1. Antibody response against HTLV-1 and Interleukin-2 (IL-2) production in the serum. BALB/c mice were inoculated with $10^{6}$ MT-2 cells intraperitoneally (IP), intravenously (IV) or perorally (PO) and sacrificed 1 month after infection. Control mice were inoculated with phosphate-buffered saline. (A) Antibody titers against HTLV-1 in the sera of mice were measured using a particle agglutination kit. (B) Splenic lymphocytes were isolated from the mice and stimulated with anti-CD3 in vitro. Production of mouse IL-2 was measured by sandwich immunoglobulin ELISA. *Mice infected IP exhibited higher IL-2 production than mice infected IV or PO or than uninfected mice (IV, $\mathrm{p}<0.01 ; \mathrm{PO}, \mathrm{p}<0.01$; control, $\mathrm{p}<0.05$; Welch's t-test).

proliferation of HTLV-1-infected cells in vivo (16). We investigated whether IL-2 production is involved in the proliferation of HTLV-1-infected cells in BALB/c mice inoculated with MT-2 cells. The mice infected intraperitoneally showed significantly higher IL-2 production than the mice infected intravenously or perorally, or than the uninfected mice (Fig. 1B).

Exclusion of MT-2 cells in inoculated mice. To determine HTLV-1 proliferation in animals inoculated with HTLV-1infected cells, the inoculated cells must be distinguished from newly infected host cells. Therefore, we previously cloned and sequenced the cellular DNA sequence flanking the 3' LTR of the HTLV-1 provirus in the MT-2 cells and established a PCR method to specifically amplify the human sequence flanking the 3' LTR of HTLV-1 provirus in the MT-2 cells (9). As shown in Fig. 2A, the MT-2-specific sequence was detected in a mouse inoculated with MT-2 cells intraperitoneally and sacrificed $12 \mathrm{~h}$ after infection, while this sequence was not detected in C91/PL cells (17), another HTLV-1-infected human cell line having different proviral integration sites, nor in the spleen of an uninfected control mouse, confirming the specificity of the MT-2-based primers. As expected, the MT-2 sequence was not detected in any of the mice 1 month after infection (Fig. 2B), consistent with previous studies showing that MT-2 cells cannot proliferate in various mouse
A

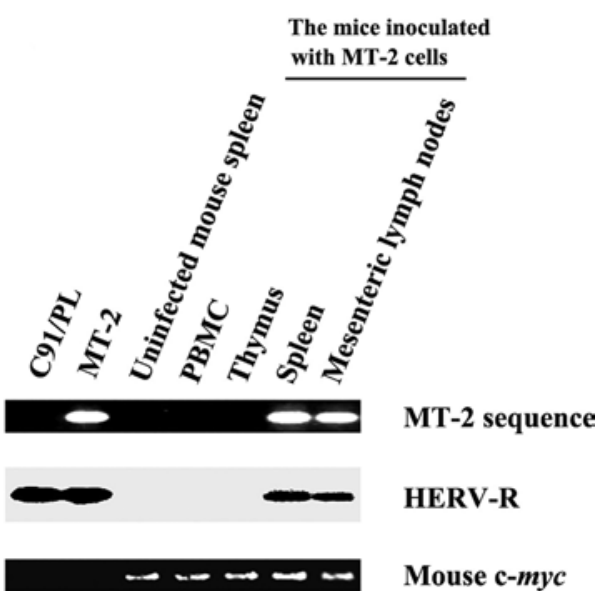

B

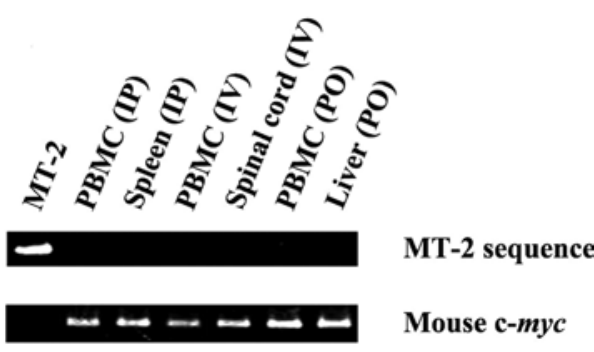

Figure 2. Detection of MT-2 genomic sequences in mice. (A) Four-week-old $\mathrm{BALB} / \mathrm{c}$ mice were inoculated with $10^{7} \mathrm{MT}-2$ cells intraperitoneally and sacrificed $12 \mathrm{~h}$ after infection. DNA was isolated from peripheral blood mononuclear cells (PBMC), thymus, spleen and mesenteric lymph nodes, and subjected to PCR amplification using primers specific for the sequence flanking the 3'LTR of the HTLV-1 provirus in human MT-2 cells, followed by agarose gel electrophoresis. To verify template DNA, control PCR amplification reactions were performed using primers specific for human endogenous retrovirus-R (HERV-R) and mouse $c$-myc. The HERV-R amplification products were verified by Southern blot analysis using a digoxigenin-labeled HERV-R-specific probe. (B) BALB/c mice were inoculated with MT-2 cells intraperitoneally (IP), intravenously (IV) and perorally (PO) and sacrificed 1 month after infection. Analysis of MT-2specific and control DNA sequences was performed as in A. Samples were subjected to agarose gel electrophoresis, and a representative image is shown.

strains containing natural killer activity, including SCID mice $(9,10,18,19)$. Together, these results indicated that the MT-2 cells were rejected in $\mathrm{BALB} / \mathrm{c}$ mice 1 month after infection, and that the proviral load in these mice represented HTLV-1infected mouse cells.

\section{Discussion}

Difficulty in obtaining human specimens has hindered the understanding of the mechanisms involved in HTLV-1 proliferation in asymptomatic carriers. Therefore, to understand viral transmission, host immune responses and the relationship between genetic background and initial viral proliferation, it is essential to establish animal models for HTLV-1 infection. A variety of animal models of HTLV-1 infection have been developed such as monkey, rabbit, rat and hamster (20). However, since mice are better characterized, particularly with respect to genetic information, and are more easily maintained than the aforementioned animal models, they are most commonly used. Previous studies examining the inoculation of immunodeficient mice, such as SCID or NOD/SCID/gammac null mice, 
with ATL cells or HTLV-1-infected cell lines, have demonstrated that these mouse models are useful for characterizing HTLV-1-transformed cells and for screening drugs for ATL patients in vivo $(21,22)$. However these models might not be suitable for studying host-virus interactions.

A few studies have compared initial routes of infection with the extent of HTLV-1 proliferation, but the effects of the routes of viral exposure differ depending on the animal model. In this report, we introduced MT-2 cells into BALB/c mice and demonstrated that intraperitoneal infection resulted in higher proviral loads compared with intravenous or peroral viral infection. In rabbits, injection of the HTLV-1 molecular clone K30p into muscle resulted in systemic HTLV-1 infection, but no provirus was detected in nervous tissue (23). It was found that the frequencies of provirus detection in peripheral blood were comparable in rats inoculated with MT-2 cells orally, intravenously or intraperitoneally (14). Another report using rats demonstrated that oral inoculation of rats with $5 \times 10^{6}$ MT-2 cells resulted in higher proviral loads compared with rats infected intraperitoneally, although proviral loads were similar among rats inoculated orally or intraperitoneally with $5 \times 10^{1}$ MT-2 cells (24). The factors controlling HTLV-1 proliferation in response to different routes of viral exposure remain unknown; however, viral or proviral loads differ greatly among the mouse strains used in retroviral infection studies (9), and endogenous retroviruses appear to influence retroviral proliferation $(25,26)$. We assessed the effects of the initial viral amount and the routes of HTLV-1 infection in mice, and HTLV-1 provirus was detected only in the spinal cord via intravenous infection (Table I). Osame et al reported that blood transfusion was associated with the onset of neurological symptoms of HAM/TSP (27). These results suggest that host-virus interactions, host immune responses, and interference from endogenous host retroviruses likely influence HTLV-1 proliferation after viral exposure through various routes of inoculation, consistent with the different levels of cytokine production observed among the infected mice in the present study (Fig. 1).

To better understand HTLV-1 proliferation shortly after viral infection via the primary routes of transmission, we inoculated mice with MT-2 cells intravenously and orally. Although we found that HTLV-1 was transmitted to the mice through both intravenous and oral routes, low proviral loads were observed 1 month after infection. In humans, transmission of HTLV-1-infected cells occurs via various cellular components, such as prostaglandins, lactoferrin, and transforming growth factor- $\beta$, found in breast milk and serum. These compounds enhance expression of glucose transporter-1 and the HTLV-1 receptor, and activate the HTLV-1 long-terminal repeat promoter (28-30). Since we inoculated $\mathrm{BALB} / \mathrm{c}$ mice with MT-2 cells suspended in phosphate-buffered saline, it is possible that efficient HTLV-1 proliferation following intravenous or oral inoculation failed owing to the absence of factors known to enhance transmission and replication of HTLV-1.

In conclusion, HTLV-1 carrier mouse models are useful for testing various anti-HTLV-1 therapeutic approaches. Furthermore, these findings have expanded the understanding of the role of viral gene products and host factors that determine HTLV-1-related pathogenesis.

\section{Acknowledgements}

This work was supported in part by a Grant-in-Aid for Cancer Research from the Ministry of Health, Labour and Welfare, Japan. T.N. was supported by individual awards from the Yasuda Medical Research Foundation and the Ishidu Shun Memorial Scholarship. M.T. and B.S. were supported in part by the Japan Society for the Promotion of Science fellowship program.

\section{References}

1. Uchiyama T: Human T cell leukemia virus type I (HTLV-I) and human diseases. Annu Rev Immunol 15: 15-37, 1997.

2. Grant C, Barmak K, Alefantis T, Yao J, Jacobson S and Wigdahl B: Human T cell leukemia virus type I and neurologic disease: events in bone marrow, peripheral blood, and central nervous system during normal immune surveillance and neuroinflammation. J Cell Physiol 190: 133-159, 2002.

3. Okochi K, Sato $\mathrm{H}$ and Hinuma Y: A retrospective study on transmission of adult $\mathrm{T}$ cell leukemia virus by blood transfusion: seroconversion in recipients. Vox Sang 46: 245-253, 1984.

4. Okayama A, Stuver S, Matsuoka M, Ishizaki J, Tanaka G, Kubuki Y, Mueller N, Hsieh CC, Tachibana N and Tsubouchi H: Role of HTLV-1 proviral DNA load and clonality in the development of adult T-cell leukemia/lymphoma in asymptomatic carriers. Int J Cancer 110: 621-625, 1984.

5. Matsuzaki T, Nakagawa M, Nagai M, Usuku K Higuchi I, Arimura K, Kubota H, Izumo S, Akiba S and Osame M : HTLV-I proviral load correlates with progression of motor disability in HAM/TSP: analysis of $239 \mathrm{HAM}$ /TSP patients including 64 patients followed up for 10 years. J Neurovirol 7: 228-234, 2001.

6. Taylor GP, Tosswill JH, Matutes E, Daenke S, Hall S, Bain BJ, Davis R, Thomas D, Rossor M, Bangham CR and Weber JN: Prospective study of HTLVI infection in an initially asymptomatic cohort. J Acquir Immune Defic Syndr 22: 92-100, 1999.

7. Hisada M, Okayama A, Shioiri S, Spiegelman DL, Stuver SO and Mueller NE: Risk factors for adult T-cell leukemia among carriers of human T-lymphotropic virus type I. Blood 92: 3557-3561, 1998.

8. Okayama A and Stuver SO: Long-term follow-up of HTLV-1 carriers. Gann Monogr Cancer Res 50: 127-139, 2003.

9. Nitta T, Tanaka M, Sun B, Hanai S and Miwa M: The genetic background as a determinant of human T-cell leukemia virus type 1 proviral load. Biochem Biophys Res Commun 309: 161-165, 2003.

10. Nitta T, Tanaka M, Sun B, Sugihara E, Kimura M, Kamada Y, Takahashi H, Hanai S, Jiang SW, Fujisawa J and Miwa M: Reduction of human T-cell leukemia virus type-1 infection in mice lacking nuclear factor-kappaB-inducing kinase. Cancer Sci 99: 872-878, 2008

11. Tanaka M, Sun B, Fang J, Nitta T, Yoshida T, Kohtoh S, Kikukawa H, Hanai S, Uchida K and Miwa M: Human T-cell leukemia virus type 1 (HTLV-1) infection of mice: proliferation of cell clones with integrated HTLV-1 provirus in lymphoid organs. J Virol 75: 4420-4423, 2001.

12. Fang J, Kushida S, Feng R, Tanaka M, Kawamura T, Abe H, Maeda N, Onobori M, Hori M, Uchida K and Miwa M: Transmission of human T-cell leukemia virus type 1 to mice. J Virol 72: 3952-3957, 1998.

13. Miyoshi I, Kubonishi I, Yoshimoto S, Akagi T, Ohtsuki Y, Shiraishi $Y$, Nagata $K$ and Hinuma Y: Type $C$ virus particles in a cord T-cell line derived by co-cultivating normal human cord leukocytes and human leukaemic T cells. Nature 294: 770-771, 1981.

14. Kato H, Koya Y, Ohashi T, Hanabuchi S, Takemura F, Fujii M, Tsujimoto H, Hasegawa A and Kannagi M: Oral administration of human T-cell leukemia virus type 1 induces immune unresponsiveness with persistent infection in adult rats. J Virol 72: 7289-7293, 1998.

15. Persaud D, Munoz JL, Tarsis SL, Parks ES and Parks WP: Time course and cytokine dependence of human T-cell lymphotropic virus type 1 T-lymphocyte transformation as revealed by a microtiter infectivity assay. J Virol 69: 6297-6303, 1995. 
16. Satoh M, Toma H, Sugahara K, Etoh K, Shiroma Y, Kiyuna S, Takara M, Matsuoka M, Yamaguchi K, Nakada K, Fujita K, Kojima S, Hori E, Tanaka Y, Kamihira S, Sato Y and Watanabe T: Involvement of IL-2/IL-2R system activation by parasite antigen in polyclonal expansion of CD4(+)25(+) HTLV-1-infected T-cells in human carriers of both HTLV-1 and S. stercoralis. Oncogene 21: 2466-2475, 2002.

17. Popovic M, Lange-Wantzin G, Sarin PS, Mann D and Gallo RC: Transformation of human umbilical cord blood $\mathrm{T}$ cells by human T-cell leukemia/lymphoma virus. Proc Natl Acad Sci USA 80 5402-5406, 1983.

18. Imada K, Takaori-Kondo A, Akagi T, Shimotohno K, Sugamura K, Hattori T, Yamabe H, Okuma M and Uchiyama T: Tumorigenicity of human T-cell leukemia virus type I-infected cell lines in severe combined immunodeficient mice and characterization of the cells proliferating in vivo. Blood 86: 2350-2357, 1995.

19. Ishihara S, Tachibana N, Okayama A, Murai K, Tsuda K and Mueller N: Successful graft of HTLV-I-transformed human T-cells (MT-2) in severe combined immunodeficiency mice treated with anti-asialo GM-1 antibody. Jpn J Cancer Res 83: 320-323, 1992.

20. Lairmore MD, Silverman L and Ratner L: Animal models for human T-lymphotropic virus type 1 (HTLV-1) infection and transformation. Oncogene 24: 6005-6015, 2005.

21. Dewan MZ, Terashima K, Taruishi M, Hasegawa H, Ito M, Tanaka Y, Mori N, Sata T, Koyanagi Y, Maeda M, Kubuki Y, Okayama A, Fujii M and Yamamoto N: Rapid tumor formation of human T-cell leukemia virus type 1-infected cell lines in novel NOD-SCID/gammac(null) mice: suppression by an inhibitor against NF-kappaB. J Virol 77: 5286-5294, 2003

22. Ohsugi T, Horie R, Kumasaka T, Ishida A, Ishida T, Yamaguchi K, Watanabe T, Umezawa $\mathrm{K}$ and Urano T: In vivo antitumor activity of the NF-kappaB inhibitor dehydroxymethyl-epoxyquinomicin in a mouse model of adult T-cell leukemia. Carcinogenesis 26 : $1382-1388,2005$
23. Simpson RM, Zhao TM, Schmidt HB, Said W and Kindt TJ: Source and route of exposure influence infectivity of a molecular clone of human T cell leukemia virus type I. AIDS Res Hum Retroviruses 14: 711-715, 1998.

24. Hasegawa A, Ohashi T, Hanabuchi S, Kato H, Takemura F, Masuda T and Kannagi M: Expansion of human T-cell leukemia virus type 1 (HTLV-1) reservoir in orally infected rats: inverse correlation with HTLV-1-specific cellular immune response. J Virol 77: 2956-2963, 2003.

25. Jude BA, Pobezinskaya Y, Bishop J, Parke S, Medzhitov RM, Chervonsky AV and Golovkina TV: Subversion of the innate immune system by a retrovirus. Nat Immunol 4: 573-578, 2003.

26. Panoutsakopoulou V, Little CS, Sieck TG, Blankenhorn EP and Blank KJ: Differences in the immune response during the acute phase of $\mathrm{E}-55^{+}$murine leukemia virus infection in progressor BALB and long term nonprogressor C57BL mice. J Immunol 161: 17-26, 1998.

27. Osame M, Janssen R, Kubota H, Nishitani H, Igata A, Nagataki S, Mori M, Goto I, Shimabukuro H and Khabbaz R: Nationwide survey of HTLV-1-associated myelopathy in Japan: association with blood transfusion. Ann Neurol 28: 50-56, 1990

28. Jones KS, Akel S, Petrow-Sadowski C, Huang Y, Bertolette DC and Ruscetti FW: Induction of human T cell leukemia virus type I receptors on quiescent naive T lymphocytes by TGF-beta. J Immunol 174: 4262-4270, 2005.

29. Moriuchi M and Moriuchi H: A milk protein lactoferrin enhances human $\mathrm{T}$ cell leukemia virus type I and suppresses HIV-1 infection. J Immunol 166: 4231-4236, 2001.

30. Moriuchi $\mathrm{M}$ and Moriuchi $\mathrm{H}$ : Transforming growth factor-beta enhances human T-cell leukemia virus type I infection. J Med Virol 67: 427-430, 2002. 